\title{
FÓRMULAS DE QUADRATURA DE CHEBYSHEV \\ E DO TIPO CHEBYSHEV
}

\author{
Marisa Veiga
}

orientador Prof. Dr. A. Sri Ranga

Dissertação apresentada ao ICMSC - USP como parte dos requisitos para obtenção do título de Mestre em ciências de computaçāo e Matemática computacional.

São Carlos - 1992 
ao prof. Ranga,

ao Jorge

e

a meus pais 


\section{Agradecimentos}

Ao prof. A. Sri Ranga, pela amizade, pela felicidade de concretizar este trabalho e de poder presenciar tantos exemplos de sabedoria, disposição e incentivos constantes.

À profa. Eliana X. L. de Andrade, pelo ótimo convívio, por todas as vezes que prontamente atendeu às minhas dúvidas e pelas inúmeras discussões muito importantes para a realização deste trabalho.

Aos meus pais, ao meu irmão Marco e a D. Maria pelo carinho e confiança.

Ao Jorge, pelo carinho, companheirismo e confiança dedicados a mim desde que nos conhecemos.

À todos os professores do DCCE - IBILCE - UNESP - São José do Rio preto, ICMSC - USP - São Carlos e do Depto de Físico QUIMICA do IQ - UNESP - Araraquara pelo apoio e incentivo.

A todos os meus amigos, em especial a Helenice e ao Kazuo.

À CAPES - Coordenação de Aperfeiçoamento de Pessoal de Nível Superior - pelo apoio financeiro.

A todos que direta ou indiretamente colaboraram para a realização deste trabalho. 


\section{RESUMO}

o objetivo deste trabalho é estudar e apresentar funçōes pesos que admitem fórmulas de quadratura de Chebyshev e do Tipo Chebyshev.

Serão apresentados, além de algumas contribuições interessantes sobre esse assunto, métodos matemáticos existentes para a construção das fórmulas e a verificação da não existência das mesmas.

Apresentamos, ainda, os estudos feitos sobre as fórmulas de quadratura do Tipo Chebyshev, com $w(x)=1$. 


\begin{abstract}
The object of this work is to study the weights functions that admit Tchebyshev quadrature rules and Tchebyshev type quadrature rules.

Apart from presenting some of the most interesting contribuitions in this topic, we give here some of the known methods for the construction of these formulas and for the verification of existence of these formulas, for any given weight function.

We give also some of the studies that have been made regarding the Tchebyshev type rule associated with $w(x)=1$.
\end{abstract}


CONTEÚDO

1 INTRODUÇÃo..............................

1.1 Introdução...........................

2 Fórmulas de quAdRATURA...................

2.1 Introdução.........................4

2.2 Fórmula de quadratura de Newton-Côtes..........5

2.3 Fórmula de quadratura de Gauss..............

2.4 Fórmula de quadratura de Chebyshev...........11

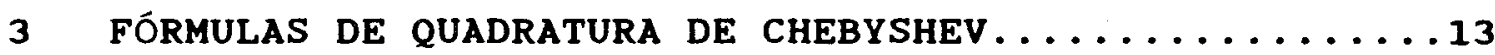

3.1 Construção da fórmula de quadratura

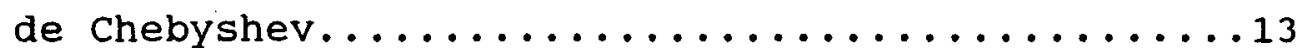

3.2 Verificação da não existência da

fórmula de quadratura de Chebyshev..........17

3.3 Casos especiais de funções pesos com

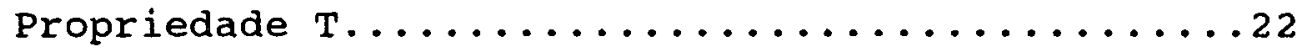

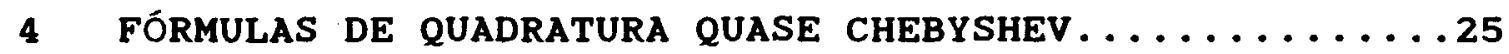

4.1 Introdução.........................25 
4.2 Construção da fórmula de quadratura

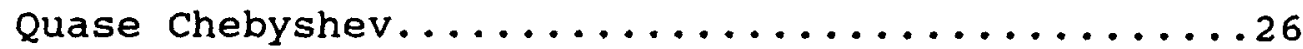

4.3 Um caso especial de função peso com

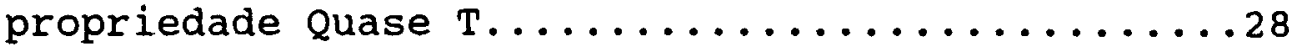

5 Fórmulas de quAdRATURA do TIPO CHEbYSHEV........... 31

5.1 Sobre fórmulas de quadratura do Tipo

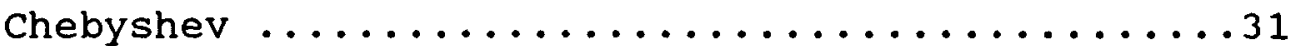

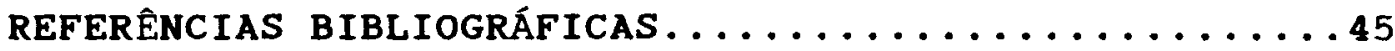


CAPÍTULO I

\section{INTRODUÇĀO}

\subsection{Introdução}

Consideremos fórmulas de quadratura do tipo

$(1.1 .1)$

$$
\int_{-1}^{+1} f(x) w(x) d x=\gamma^{(n)} \sum_{r=1}^{n} f\left(x_{r}^{(n)}\right)+R_{n}(f)
$$

onde $x_{r}^{(n)}$ são reais, distintos e pertencem ao intervalo $(-1,1)$. Aqui, $w(x)$ são funções não negativas no intervalo $[-1,1]$.

Tais fórmulas de quadratura têm a propriedade de minimizar o efeito de erros randômicos nos valores de $f\left(x_{r}\right)$, podendo ser uma característica útil se esses erros forem considerados maiores que - erro de truncamento $R_{n}(f)$.

Um outro fato desejável nessas fórmulas de quadratura é que somente uma multiplicação é exigida na avaliação da soma (1.1.1), sem contar o trabalho de avaliar a função.

Se $R_{n}(f)$ satisfaz

$$
R_{n}(f)=0, \quad \text { para todo } f \in \mathbb{P}_{n} \text {, }
$$

(onde $\mathbb{P}_{n}$ denota a classe de polinômios de grau menor ou igual a $n$ ) essas fórmulas de quadratura são conhecidas por fórmulas de quadratura de Chebyshev.

Pelo fato de $R_{n}(f)=0$, para $f(x)=1$ obtemos : 


$$
\gamma^{(n)}=\frac{1}{n} \int_{-1}^{+1} w(x) d x=\frac{\mu_{0}}{n}
$$

para as fórmulas de quadratura de Chebyshev.

É bem conhecido que a clássica função peso $w(x)=\left(1-x^{2}\right)^{-1 / 2}$ admite quadratura de Chebyshev para todo n $\geq 1$. Funções pesos que admitem a fórmula de quadratura de Chebyshev para todo $n \geq 1$ são conhecidas por funçōes pesos com propriedade $T$. Tais funções são consideradas raras. Estudos recentes para se achar e caracterizar classes de funçōes pesos com propriedade $T$, estão sendo feitos por vários pesquisadores. o objetivo desta dissertação é estudar as fórmulas de quadratura de chebyshev e as fórmulas de quadratura do Tipo Chebyshev com grau de precisão dado por
$(1.1 .4)$
$R_{n}(f)=0$,
para todo $f \in \mathbb{P}_{p}$,
com $p \leq n-1$

Em particular, consideramos as fórmulas de quadratura com $\mathrm{p}=\mathrm{n}-1$, as quais nós chamaremos de fórmulas de quadratura "Quase" Chebyshev.

Apresentamos algumas funções pesos que admitem fórmulas de quadratura de Chebyshev para todo $n \geq 1$. Desenvolvemos também um caso especial de função peso que fornece a fórmula de quadratura Quase Chebyshev com propriedades desejáveis.

Para isso dividimos o trabalho em 5 capitulos:

No capitulo 2 - Fórmulas de Quadratura - apresentamos o desenvolvimento histórico das fórmulas de quadratura, desde as fórmulas de quadratura de Newton-Côtes até as de Chebyshev. 
No capítulo 3 - Fórmulas de Quadratura de Chebyshev apresentamos técnicas matemáticas para a construção da quadratura de Chebyshev e para a verificação da sua não existência. Veremos também algumas funções pesos com propriedade T, isto é, funções pesos que admitem fórmula de quadratura de chebyshev para todo $n \geq 1$.

No capítulo 4 - Fórmulas de Quadratura Quase Chebyshev apresentamos uma técnica para a construção dessas fórmulas e desenvolvemos um caso especial de função peso que admite essa fórmula para todo $n \geq 1$.

No capítulo 5 - Fórmulas de Quadratura do Tipo Chebyshev apresentamos os estudos que foram feitos sobre as fórmulas de quadratura do Tipo Chebyshev $\operatorname{com} \mathrm{w}(\mathrm{x})=1$ no intervalo $[-1,1]$.

Por último, nas Referências Bibliográficas relacionamos os livros e artigos por nós consultados. 


\section{FÓRMULAS DE QUADRATURA}

\subsection{Introdução}

A história das fórmulas de quadratura iniciou-se com Newton e Côtes. Newton, em 1676, foi o primeiro a sugerir, verdadeiramente, um método geral para aproximação de integrais. Côtes, independentemente, chegou a um método semelhante.

Em 1814, Gauss utilizou o trabalho de Newton e Côtes como ponto de partida, combinou-o com seu próprio trabalho sobre séries hipergeométricas, e desenvolveu seu famoso método para integração que melhorou significativamente o método de Newton e Côtes. O trabalho de Gauss foi simplificado por Jacobi e, durante - século XIX, Mehler, Christoffel e outros pesquisadores desenvolveram-no bastante.

Ainda neste século, Chebyshev começou seu estudo sobre quadratura, por volta de 1874 .

Grandes progressos foram feitos depois por Bernsteint[2]t, Ullman [25], Geronimus [12] e outros. 


\section{2 Fórmula de Quadratura de Newton e Côtes}

Em um de seus primeiros trabalhos, Newton mostrou que :

Dada uma função $f$ e $n$ pontos distintos $x_{r}$, é possivel construir um único polinômio $p_{n-1}(f)$, de grau menor ou igual a $n-1$, que coincide com a função $f$ nos pontos $x_{r}$, ou seja

$$
p_{n-1}\left(f, x_{r}\right)=f\left(x_{r}\right) \quad r=1,2, \ldots, n, \quad p_{n-1} \in \mathbb{P}_{n-1} .
$$

Newton, engenhosamente, expressou esta interpolação polinomial em termos de diferenças divididas. Aqui achamos conveniente expressar na forma dada por Lagrange em 1795,

$$
p_{n-1}(f, x)=\sum_{r=1}^{n} l_{r}(x) f\left(x_{r}\right)
$$

onde $l_{r}(x)$ são polinômios especiais de grau $n-1$ com $1_{r}\left(x_{r}\right)=1$ e $l_{r}\left(x_{u}\right)=0, u \neq x$. Se escrevermos

$$
f(x)=p_{n-1}(f, x)+r_{n}(x)
$$

onde $r_{n}(x)$ denota o erro na interpolação, temos, então, pela unicidade do polinômio interpolador que

$$
r_{n}(x)=0 \text { para todo } f \in \mathbb{P}_{n-1} \text {. }
$$

Se

$$
I(f)=\int_{a}^{b} f(x) d x,
$$

onde $a<b$ são finitos, $a$ integração de (2.2.2) fornece
$(2 \cdot 2 \cdot 5)$
$I(f)=Q_{n}(f)+R_{n}(f)$,
$Q_{n}(f)=\sum_{r=1}^{n} \lambda_{r} f\left(x_{r}\right)$ 
onde, por $(2.2 .1)$

$$
\lambda_{r}=I\left(1_{r}\right), \quad r=1,2, \ldots, n,
$$

$(2.2 .6)$

$$
R_{n}(f)=I\left(r_{n}(x)\right)
$$

los coeficientes $\lambda_{r}$, tanto quanto os pontos $x_{r}$, dependem de $n$, mas para simplificar suprimimos esta dependência na notação.) Chamaremos (2.2.5) de fórmula de quadratura para $n$ pontos, $Q_{n}$ (f) a soma da quadratura, e $R_{n}(f)$ o resto. Os pontos $x_{r}$ são chamados nós enquanto que os números $\lambda_{r}$ são os pesos para a fórmula de quadratura. A soma $Q_{n}(f)$ é "esperada" aproximar a integral.

Como consequência de $(2.2 .3)$,

$$
R_{n}(f)=0 \text { para todo } f \in \mathbb{P}_{n-1} \text {. }
$$

Em virtude de (2.2.7), a regra de quadratura expressa por $Q_{n}(f)$ é dita ter grau de precisão algébrica n-1. Radau [22] foi o primeiro a usar esta expressão. Usaremos aqui $d\left(Q_{n}\right)=n-1$. fácil ver que a fórmula de quadratura $Q_{n}(f)$ tem grau de precisão $n-1$ se e somente se é obtida por meio de interpolação, como descrito acima. Portanto, $Q_{n}(f)$, para o qual (2.2.7) é satisfeita, é também chamada quadratura interpolatória.

No caso de pontos $x_{r}, r=1,2, \ldots, n$ igualmente espaçados, os números $\lambda_{r}$ em $(2.2 .6)$ podem ser calculados todos de uma só vez. Tais números são chamados números de côtes, que foi o primeiro a obtê-los para $\mathrm{n}$ até 11 .

Fórmulas de quadratura interpolatória com nós igualmente 
espaçados também são chamadas fórmulas de quadratura de Newton-côtes. Esta inclui, como casos especiais a fórmula do Trapézio e fórmula de simpson.

No caso de nós arbitrários (distintos), $x_{r}$, os números $\lambda_{r}$ podem ser expressos em termos do polinômio nodal

$$
w_{n}(x)=\prod_{r=1}^{n}\left(x-x_{r}\right) \text {. }
$$

Com (2.2.6) usando a fórmula de Lagrange para $1_{r}$, obtemos:

$$
\lambda_{r}=I\left[\frac{W_{n}(x)}{W_{n}^{\prime}\left(x_{r}\right)\left(x-x_{r}\right)}\right], r=1,2, \ldots, n .
$$

\subsection{Fórmula de Quadratura de Gauss}

Suponhamos que os nós $x_{r}$ da fórmula de quadratura interpolatória sejam distintos e variam livremente. Qual será o máximo grau de precisão que pode ser atingida ? Como selecionar os nós $x_{r}$ para se realizar está otimização ?

Estas foram questões levantadas por Gauss [7], e respondidas elegantemente por meio da teoria de frações contínuas associadas a séries hipergeométricas.

Gauss conjecturou que $\max _{x_{r}, \lambda} d\left(Q_{n}\right)=2 n-1$ uma vez existem $2 n$ incógnitas a serem encontradas, e $2 \mathrm{n}$ condiçōes impostas. Para verificar a conjectura, Gauss partiu do erro na integração, $R_{n}(f)$, para $f$ igual à função característica $1 / z-t$ 


$$
R_{n}\left(\frac{1}{z-t}\right)=\sum_{k=0}^{\infty} \frac{R_{n}\left(t^{k}\right)}{z^{k+1}}
$$

o problema, então, equivale a determinar $x_{r}, \lambda_{r}$ tal que

$$
R_{n}\left(\frac{1}{z-t}\right)=0\left[1 /\left(z^{2 n+1}\right)\right], z-\infty
$$

Observamos que para a integral em (2.2.4), quando $\mathbf{b}=-\mathbf{a}=1$, temos

$$
I\left(\frac{1}{z-t}\right)=\ln \left(\frac{1+1 / z}{1-1 / 2}\right)=\frac{2}{z-} \frac{a_{2}}{2-} \frac{a_{3}}{z-} \ldots,
$$

$$
\text { onde } \quad a_{n}=\frac{(n-1)^{2}}{\prod_{r=1}^{n}(2 r-1)}, \quad n \geq 2
$$

A fração contínua da expressão acima, era bem conhecida de Gauss [6]. Ele também conhecia que o n-ésimo convergente, uma função racional $R_{n-1, n}$ com numerador de grau n-1 e denominador de grau $n$, se expandida em potências recíprocas de $z$, aproxima a função que está à esquerda, até a ordem de $z^{-2 n-1}$. Isto é,

$$
I\left(\frac{1}{z-t}\right)=R_{n-1, n}(z)+O\left[1 /\left(z^{2 n+1}\right)\right], z-\infty
$$

Gauss, agora, decompos $R_{n-1, n}$ em frações parciais e tomou os resíduos e polos como os pesos e nós da fórmula de quadratura $(2.2 .5)$. Isto é,

$$
R_{n-1, n}(z)=\sum_{r=1}^{n} \frac{\lambda_{r}}{z-x_{r}}=Q_{n}\left(\frac{1}{z-t}\right) \text {. }
$$

Daŕ, então, segue que 


$$
\begin{aligned}
R_{n}\left(\frac{1}{z-t}\right) & =I\left(\frac{1}{z-t}\right)-Q_{n}\left(\frac{1}{z-t}\right) \\
& =I\left(\frac{1}{z-t}\right)-R_{n-1, n}(z) \\
& =0\left[1 /\left(z^{2 n+1}\right)\right], \quad z \sim \infty
\end{aligned}
$$

e, consequentemente $d\left(Q_{n}\right)=2 n-1$ como desejado.

A descoberta de Gauss foi considerada como um dos eventos mais significativos de século XIX na área de integração numérica e talvez em toda a Análise Numérica.

- resultado tem, não apenas grande beleza, mas também teve grande influência em muitos resultados futuros em computação e aproximação.

Logo incentivou comtemporâneos, tais como Jacobi e Christoffel, a aperfeiçoar o método de Gauss e desenvolvê-lo em novas direções.

A fração contínua em (2.3.1) e sua associação próxima com a integral $I[1 /(z-x)]$ foi vista por Gauss como a verdadeira fonte de seu novo método de integração. Jacobi [14], por outro lado, com clareza e simplicidade característica deduziu o resultado de Gauss baseando-se puramente em argumentos de divisibilidade de polinômios.

o conceito central que surgiu do trabalho de Jacobi foi a ortogonalidade.

De fato, Jacobi mostra que, dado qualquer inteiro $k$, com $0 \leq k \leq n$, a regra de quadratura $Q_{n}$ em (2.2.5) tem grau de precisão $n-1+k$ se, e somente se, as seguintes condições são satisfeitas : 

(i) $Q_{n}$ é interpolatória ;
(ii) $I\left(w_{n} p\right)=0$, para todo $p \in \mathbb{P}_{k-1}$;

onde $w_{n}$ é o polinômio dado por (2.2.8). A condição (ii) requer que $w_{n}$ seja ortogonal a todos os polinômios de grau menor ou igual $\mathrm{k}-1$.

- valor de $k$ deve ser menor ou igual a $n$, já que $w_{n}$ não pode ser ortogonal a ele mesmo. Em particular, se $k=n$ então é atingido o máximo grau de precisão que é 2 n-1.

o argumento de Jacobi é extremamente transparente. claramente (i) é necessário e a necessidade de (ii) é consequência de

$$
I\left(w_{n} p\right)=Q_{n}\left(w_{n} p\right) \text {, para todo polinômio } p \in \mathbb{P}_{k-1}
$$

e de $Q_{n}\left(w_{n} p\right)=0$, sendo $w_{n}$ o polinômio que se anula para todos os nós $x_{r}^{(n)}$.

Para a suficiência, seja p um polinômio arbitrário de grau menor ou igual a $n-1+k$. Dividindo $p$ por $w_{n}$ temos

$$
p=q w_{n}+r, \quad q \in \mathbb{P}_{k-1}, \quad r \in \mathbb{P}_{n-1} \text {. }
$$

$$
\begin{aligned}
& \text { Então, } I(p)=I\left(q w_{n}\right)+I(r) \\
& =I(r) \quad \text { por (ii) } \\
& =Q_{n}(r) \quad \text { por (i) } \\
& =Q_{n}(p)-Q_{n}\left(q w_{n}\right)=Q_{n}(p) \text {. }
\end{aligned}
$$

Isto é, $Q_{n}$ tem grau de precisão $n-1+k$.

Depois das contribuições de Jacobi, as fórmulas de quadratura de Gauss foram desenvolvidas de diversas maneiras, 
por muitos pesquisadores, como por exemplo, Christoffel, Mehler, etc. .

\section{4 Fórmula de Quadratura de Chebyshev}

- estudo das quadraturas de Chebyshev começou com um trabalho clássico de Chebyshev [4] por volta de 1874.

Neste trabalho, ele propôs um método para construção dessas fórmulas. Com este método conseguiu chegar, para o peso $w(x)=1 /\left(1-x^{2}\right)^{1 / 2}$ à fórmula de quadratura

$$
\int_{-1}^{+1} f(x) \frac{1}{\left(1-x^{2}\right)^{1 / 2}} d x=\frac{\pi}{n} \sum_{r=1}^{n} f\left(x_{r}\right)+R_{n}(f)
$$

onde $\quad x_{r}=\cos \left(\frac{2 r-1}{2 n} \pi\right)$ e $\quad R_{n}(f)=0$ para todo $f \in \mathbb{P}_{n}$.

Ele, por alguma razão desconhecida, atribui esta fórmula a Hermite.

Entretanto, a fórmula já tinha sido descoberta por Mehler [17], que mostrou que é uma fórmula de quadratura do tipo Gauss com $R_{n}(f)=0$ para todo $f \in \mathbb{P}_{2 n-1}$. Hoje esta fórmula de quadratura é conhecida por fórmula de quadratura de chebyshev ou simplesmente quadratura Gauss-Chebyshev. 0 peso $\left(1-x^{2}\right)^{-1 / 2}$ é conhecido por peso de Chebyshev.

Chebyshev também considerou a fórmula de quadratura de Chebyshev para o peso $\mathrm{w}(\mathrm{x})=1$. Ele, usando seu método, calculou os valores de $x_{r}$, para $n=2,3, \ldots, 7$ e mostrou que estes valores 
são reais, distintos e pertencentes ao intervalo $[-1,1]$. Esta fórmula para $w(x)=1$ é, hoje, conhecida como a fórmula clássica da quadratura de Chebyshev.

Radau [22] acrescentou à fórmula clássica de quadratura de Chebyshev o caso $n=9$, que também produz nós reais e mostrou que se $n=8$, envolve nós complexos.

Cinquenta anos depois, Bernstein [2] provou que somente as fórmulas encontradas por chebyshev e Radau tem nós reais,e se n=8 ou $n>9$, necessáriamente envolve nós complexos.

Até a descoberta de Ullman [25] em 1966, a função peso de Chebyshev $w(x)=\left(1-x^{2}\right)^{-1 / 2}$, foi a única conhecida para a qual a fórmula de quadratura tem nós reais para todo $n \geq 1$.

Desde então, vários novos resultados foram obtidos. Veremos alguns deles no próximo capítulo. 


\section{FÓRMULAS DE QUADRATURA DE CHEBYSHEV}

3. 1 Construção da Fórmula de quadratura de Chebyshev

MÉTODO DE CHEBYSHEV

Este foi o método utilizado por Chebyshev $[4]$. Ele representou o polinômio, $\mathrm{P}_{n}(z, w(x))$ cujas raizes são os nós da fórmula de quadratura, explicitamente na forma

$(3.1 .1)$

$$
\begin{aligned}
& P_{n}(z, w(x))=E\left\{[f(z)]^{n}\right\}, \text { onde } \\
f(z) & =\exp \left(\frac{1}{\mu_{0}} \int_{a}^{b} \ln (z-x) w(x) d x\right) \\
& =z \exp \left(-\frac{1}{\mu_{0}} \sum_{k=1}^{\infty} \frac{\mu_{k}}{k z^{k}}\right), \quad|z|>1
\end{aligned}
$$

e $E\{\cdot\}$ denota a parte polinomial da função $\{\cdot\}$.

Verifiquemos este resultado:

É fácil mostrar que

$$
\frac{P_{n}^{\prime}(z, w(x))}{n P_{n}(z, w(x))}=\frac{1}{n} \sum_{r=1}^{n} \frac{1}{z-x_{r}^{(n)}}
$$

onde $x_{r}^{(n)}$ são as raizes de $P_{n}(z, w(x))$.

Expandindo o lado direito da expressão acima em termos de $1 / 2$, obtemos: 


$$
\frac{P_{n}^{\prime}(z, w(x))}{n P_{n}(z, w(x))}=\sum_{k=0}^{\infty} \frac{\frac{1}{n} \sum_{r=1}^{n}\left(x_{r}^{(n)}\right)^{k}}{z^{k+1}}
$$

Por outro lado, de (3.1.1), temos:

$$
P_{n}(z, w(x))=\{f(z)\}^{n}+G_{n}(z)
$$

onde, $\quad G_{n}(z)=\frac{g_{0}^{(n)}}{z}+\frac{g_{1}^{(n)}}{z^{2}}+\frac{g_{2}^{(n)}}{z^{3}}+\ldots$ é uma função analítica para $|z|>1$, que se anula no infinito.

Derivando em relação a $z$ obtemos :

$$
\begin{aligned}
& P_{n}^{\prime}(z, W(x))=n\{f(z)\}^{n-1} f^{\prime}(z)+G_{n}^{\prime}(z), \text { com } \\
& G_{n}^{\prime}(z)=-\frac{g_{0}^{(n)}}{z^{2}}-\frac{2 g_{1}^{(n)}}{z^{3}}-\frac{3 g_{2}^{(n)}}{z^{4}}-\ldots .
\end{aligned}
$$

Segue, de $(3.1 .3)$ e $(3.1 .4)$ que

$$
\begin{gathered}
\frac{P_{n}^{\prime}(z, w(x))}{n P_{n}(z, w(x))}=\frac{f^{\prime}(z)}{f(z)}+\frac{1}{P_{n}(z, w(x)}\left\{\frac{G_{n}^{\prime}(z)}{n}-\frac{f^{\prime}(z)}{f(z)} G_{n}(z)\right\}, \\
\frac{P_{n}^{\prime}(z, w(x))}{n P_{n}(z, w(x))}=\frac{f^{\prime}(z)}{f(z)}+O\left[1 /\left(z^{n+2}\right)\right]
\end{gathered}
$$

Considerando a expansão de Laurent de $f^{\prime}(z) / f(z)$ obtemos

$$
\frac{P_{n}^{\prime}(z, w(x))}{n P_{n}(z, w(x))}=\sum_{k=0}^{n} \frac{\mu_{k} / \mu_{0}}{z^{k+1}}+O\left[1 /\left(z^{n+2}\right)\right]
$$

Comparando os primeiros $n+1$ termos de $(3.1 .2)$ e (3.1.5) temos que 


$$
\mu_{k}=\frac{\mu_{0}}{n} \sum_{r=1}^{n}\left(x_{r}^{(n)}\right)^{k}, \quad k=1, \ldots, n .
$$

Isto é,

$$
\int_{a}^{b} x^{k} w(x) d x=\frac{\mu_{0}}{n} \sum_{r=1}^{n}\left(x_{r}^{(n)}\right)^{k}, k=1, \ldots, n .
$$

Esta relação nos diz que se escolhermos $x_{r}^{(n)}$, que são os zeros de $P_{n}(z, w(x))$, para serem os nós da fórmula de quadratura (1.1.1), então a fórmula é exata para todo polinômio de grau menor ou igual a $\mathrm{n}$.

Baseado nesta fórmula, Chebyshev calculou a regra de quadratura original $(\operatorname{com} w(x)=1)$ no intervalo $(-1,1)$ para $\mathrm{n}=2,3, \ldots, 7$.

No caso de $w(x)=\left(1-x^{2}\right)^{-1 / 2}$ no intervalo $[-1,1]$, o polinômio (3.1.1) pode ser dado por

$$
\begin{gathered}
P_{n}(z, w(x))=E\left\{\left(\frac{z+\left(z^{2}-1\right)^{1 / 2}}{2}\right)^{n}\right\} \\
=\left\{\left(\frac{z+\left(z^{2}-1\right)^{1 / 2}}{2}\right)^{n}+\left(\frac{z-\left(z^{2}-1\right)^{1 / 2}}{2}\right)^{n}\right\}=\frac{1}{2^{n-1}} T_{n}(z)
\end{gathered}
$$

onde $T_{n}(z)$ é o polinômio de Chebyshev de primeira espécie. Deste modo, Chebyshev restabelece a regra de quadratura

$$
\begin{aligned}
& \int_{-1}^{+1} f(x)\left(1-x^{2}\right)^{-1 / 2} d x=\frac{\pi}{n} \sum_{k=1}^{n} f\left(x_{k}^{(n)}\right)+R_{n}(f), \\
& \text { onde } x_{k}^{(n)}=\cos \left(\frac{2 k-1}{2 n} \pi\right), \quad k=1,2, \ldots, n,
\end{aligned}
$$

que ele atribue a Hermite. 
Se a fórmula de quadratura

$(3.1 .7)$

$$
\int_{a}^{b} f(x) w(x) d x=\frac{\mu_{0}}{n} \sum_{k=1}^{n} f\left(x_{k}^{(n)}\right)+R_{n}(f)
$$

tem grau de precisão algébrica $n$, então os nós $x_{k}=x_{k}^{(n)}$ deverão satisfazer

$$
\sum_{k=1}^{n} x_{k}^{r}=\frac{n}{\mu_{0}} \mu_{r}, \quad r=1,2, \ldots, n .
$$

Desde que os momentos $\mu_{r}$ são conhecidos, estas equações determinam as $n$ primeiras somas de potência $s_{r}=\sum_{k=1}^{n} x_{k}^{r}$ para os nós.

Estas, por sua vez, determinam os coeficientes $a_{k}$ do polinômio $P_{n}(x, w(x))$ pelas identidades de Newton

$$
\begin{aligned}
& s_{1}+a_{1}=0 \\
& s_{2}+a_{1} s_{1}+2 a_{2}=0 \\
& \ldots \ldots \ldots \ldots \ldots \ldots \ldots \\
& s_{n}+a_{1} s_{n-1}+\ldots+a_{n-1} s_{1}+n a_{n}=0
\end{aligned}
$$

Os nós desejados, $x_{k}$, em (3.1.7), podem ser calculados encontrando-se as raizes da equação algébrica $x^{n}+a_{1} x^{n-1}+\ldots+a_{n}=0$, onde os coeficientes $a_{k}$, $k=1, \ldots, n$, são obtidos recursivamente de $(3.1 .8)$ e as somas $s_{r}$ são calculadas por 


$$
\mathrm{s}_{\mathrm{r}}=\frac{\mathrm{n}}{\mu_{0}} \mu_{\mathrm{r}}, \quad \mathrm{r}=1,2, \ldots \mathrm{n} .
$$

Este método, primeiramente utilizado por Radau [22], é a técnica mais comumente aplicada para calcular quadratura de Chebyshev para pesos arbitrários.

3.2 Verificação da não existência da fórmula de quadratura de Chebyshev

MÉTODO DE BERNSTEIN

Este método é considerado uma ferramenta "poderosa" para se provar resultados de não existência. Embora Bernstein [2] tenha tratado somente o caso em que a função peso é constante, este método se estende a funções pesos arbitrárias $\mathrm{w}(\mathrm{x})$.

A idéia de Bernstein é de confrontar a fórmula de quadratura de Chebyshev para $n$ pontos,

(3.2.1) $\quad \int_{a}^{b} f(x) w(x) d x=\frac{\mu_{0}}{n} \sum_{k=1}^{n} f\left(x_{k}^{(n)}\right)+R_{n}^{c h}$ (f) ,

com a fórmula de Gauss de m pontos

$(3.2 .2)$

$$
\int_{a}^{b} f(x) w(x) d x=\sum_{r=1}^{m} \lambda_{r}^{(m)} f\left(\xi_{r}^{(m)}\right)+R_{m}^{G}(f)
$$


onde $\xi_{r}^{(m)}$ são os zeros de $\pi_{m}(x, w(x))$, em ordem decrescente $a<\xi_{m}^{(m)}<\xi_{m-1}^{(m)}<\ldots<\xi_{1}^{(m)}<b$ e $\lambda_{r}^{(m)}$ são os correspondentes números de Christoffel. Supondo que os nós $x_{k}$ em (3.2.1) são distintos , todos contidos no intervalo $(a, b)$ e que a fórmula (3.2.1) seja exata para polinômios de grau menor ou igual $2 m-1$, $m<n$, Bernstein mostra que necessariamente

$$
\frac{\mu_{0}}{n} \leq \min \left(\lambda_{1}^{(m)}, \lambda_{m}^{(m)}\right)
$$

Ilustraremos a aplicação da desigualdade (3.2.3) para provar a não existência de uma fórmula de quadratura de Chebyshev.

Seja $w(x)$ a função peso de Laguerre, isto é, $w(x)=x^{\alpha} e^{-x}, \alpha>-1$.

Então, a expressão (3.2.1) tem a forma

$$
\int_{0}^{\infty} f(x) x^{\alpha} e^{-x} d x=\frac{\Gamma(\alpha+1)}{n} \sum_{k=1}^{n} f\left(x_{k}^{(n)}\right)+R_{n}(f), \quad \alpha>-1
$$

Os polinômios ortogonais são os polinômios de Laguerre normalizados

$$
\pi_{0}(x)=[\Gamma(\alpha+1)]^{-1 / 2},
$$

$$
\begin{gathered}
\pi_{1}(x)=[\Gamma(\alpha+2)]^{-1 / 2}(\alpha+1-x) \ldots \\
\lambda_{1}^{(m)}=\left(\sum_{k=0}^{m-1}\left[\pi_{k}\left(\xi_{1}^{(m)}\right)\right]^{2}\right)^{-1} \leq\left(\pi_{0}^{2}+\left[\pi_{1}\left(\xi_{1}^{(m)}\right)\right]^{2}\right)^{-1} \quad m \geq 2 .
\end{gathered}
$$

Usando uma conhecida desigualdade (Krylov [17])

$$
\xi_{1}^{(m)}>2 m+\alpha-1 \quad(m \geq 2, \alpha>-1),
$$

e a expressão dada em (3.2.5), podemos estimar 
$(3.2 .6)$

$$
\lambda_{1}^{(m)}<\frac{\Gamma(\alpha+1)}{1+4(m-1)^{2} /(\alpha+1)} \quad, \quad m \geq 2 .
$$

Suponhamos $\mathrm{n}$ par, $\mathrm{n}=2 \mathrm{~m}(\mathrm{~m} \geq 2)$ e, como a regra de quadratura (3.2.4) tem grau de precisão n, então, certamente, tem grau de precisão $2 m-1$. E, pela desigualdade de Bernstein,

$$
\frac{\Gamma(\alpha+1)}{n} \leq \lambda_{1}^{(m)}
$$

Se a desigualdade acima não for satisfeita, então a regra de quadratura $(3.2 .4)$ é impossível. Em virtude de (3.2.6) será este o caso se

$$
\frac{1}{n} \geq \frac{1}{1+4(m-1)^{2} /(\alpha+1)} \quad, m \geq 2 \text {. }
$$

Desde que $n=2 m$, a última desigualdade equivale a

$$
n^{2}-(\alpha+5) n+\alpha+5 \geq 0, \quad n \geq 4, \text { ou seja, a }
$$

$$
\mathrm{n} \geq \frac{1}{2}\left\{\alpha+5+((\alpha+1)(\alpha+5))^{1 / 2}\right\} \text { e } n \geq 4
$$

Portanto, para todo $\mathrm{n}$ par satisfazendo (3.2.8), a fórmula (3.2.4) não existe. Um argumento semelhante pode ser aplicado para n impar, e, também, para quadraturas do tipo chebyshev (3.2.4), para um dado grau de precisão menor que $n$ (Gautschi [8]).

\section{MÉtodos BASEAdOS EM SEQUÊNCIAS DE MOMENTOS}

Observamos em (3.1.9) que, para (3.1.7) ser uma fórmula de 
quadratura de Chebyshev, é necessário e suficiente que os nós sejam reais e que

$$
s_{r}=\frac{n}{\mu_{0}} \mu_{r^{\prime}} \quad r=1,2, \ldots, n \text {, }
$$

onde $s_{r}=\sum_{k=1}^{n} x_{k}^{r}$ são as somas de potência nos nós $x_{k}=x_{k}^{(n)}$.

Assim, qualquer propriedade válida para somas de potência $\mathbf{s}_{\mathbf{r}}$ em variáveis reais imediatamente se traduzem em uma propriedade para os momentos $\mu_{r}, r=1,2, \ldots, n, o$ que, por sua vez representam uma condição necessária para (3.1.7) ser uma regra de quadratura de Chebyshev. A violação dessa propriedade implica na não existência de (3.1.7). Uma dessas propriedades, usada por wilf [26], é a desigualdade de Jensen, que afirma que para números não negativos, $\xi_{k} \geq 0$, as quantidades $\sigma_{r}=\left(\sum_{k=1}^{n} \xi_{k}^{r}\right)^{1 / r}$ são não crescentes em $r$ para $r>0$, isto é, $\sigma_{r} \geq \sigma_{s}$, sempre que $0<\mathrm{r}<\mathrm{s}$ (Hardy, Littlewood e Pólya [13]).

$$
\text { Consequentemente, se todo } x_{k} \geq 0 \text {, então }
$$

$$
\tau_{r}=\left(\frac{n}{\mu_{0}} \mu_{r}\right)^{1 / r} \text { é não crescente para } r=1,2, \ldots, n \text {, }
$$

e, se todos os $x_{k}$ são reais arbitrários, $k=1,2, \ldots, n$,

$$
\tau_{r}^{*}=\left(\frac{\mathrm{n}}{\mu_{0}} \mu_{2 r}\right)^{1 / r} \text { é não crescente, }
$$

para $r=1,2, \ldots,[n / 2]$.

Tureckii [24] e, mais tarde, Janovic [15] e Nutfullin e Janovic [20] usam as desigualdades 


$$
\begin{array}{ll}
s_{n} \leq s_{n-2 r} s_{2 r} & (n \text { par }, r=1,2, \ldots, n / 2), \\
s_{n-1} \leq s_{n-1-2 r} s_{2 r} & (n \text { impar, } r=1,2, \ldots,(n-1) / 2) .
\end{array}
$$

válidas para valores arbitrários $\mathbf{x}_{\mathbf{k}}$, para obter as condições necessárias

$$
\frac{\mu_{n}}{n \mu_{n-2 r}} \leq \frac{\mu_{2 r}}{\mu_{0}} \quad(n \text { par ; r }=1,2, \ldots, n / 2),
$$

$(3.2 .11)$

$$
\frac{\mu_{n-1}}{n \mu_{n-1-2 r}} \leq \frac{\mu_{2 r}}{\mu_{0}} \quad(n \text { ímpar ; } r=1,2, \ldots,(n-1) / 2) \text {. }
$$

Para ilustrar o uso de (3.2.9), consideremos novamente a função peso de Laguerre $x^{\alpha} e^{-x}, \alpha>-1$, para a qual $\mu_{r}=\Gamma(\alpha+r+1), r=0,1,2, \ldots$. Exigindo nós $x_{k}$, reais e não negativos, podemos aplicar (3.2.9), isto é, $\tau_{r-1} \geq \tau_{r}$ para $2 \leq r \leq n$, que, para $r=n$ fornece

$$
\left(\frac{n}{\mu_{0}} \mu_{n-1}\right)^{1 / n-1} \geq\left(\frac{n}{\mu_{0}} \mu_{n}\right)^{1 / n},
$$

ou , equivalentemente,

$$
\frac{n}{\Gamma(\alpha+1)} \frac{\Gamma(\alpha+n+1)}{(\alpha+n)^{n}} \geq 1 \text {. }
$$

Desde que o lado esquerdo é assíntoticamente igual a $(2 \pi)^{1 / 2} e^{-\alpha}[\Gamma(\alpha+1)]^{-1} n^{\alpha+3 / 2} e^{-n}$, quando $n \rightarrow \infty$, é claro que (3.2.12) será falso para todo $\mathrm{n}$ suficientemente grande, portanto a quadratura de Chebyshev não é possível.

Analogamente, fazendo $r=1$ na primeira equação de 
Analogamente, fazendo $r=1$ na primeira equação de (3.2.11) encontramos a condiçāo necessária (Tureckii [24])

$$
n^{2}-\left(\alpha^{2}+\alpha+3\right) n+\alpha(\alpha-1) \leq 0, n \geq 4 \text { (par), }
$$

que leva a uma condição de não existência semelhante àquela obtida pelo método de Bernstein em (3.2.8).

\subsection{Casos especiais de funções pesos com Propriedade T}

A questão da construção das fórmulas de quadratura de Chebyshev é trabalhosa. A principal tarefa é a de calcular os zeros do polinômio que proporcionam os nós. Tais zeros, entretanto, podem ser muito difíceis de se obter, particularmente se a função peso dada contém parâmetros.

Como vimos, uma função peso bem conhecida com propriedade $T$ é a função peso $w_{0}(x)=\left(1-x^{2}\right)^{-1 / 2}$ de Chebyshev. Esta função peso foi a única conhecida até 1966, quando Ullman [25] apresentou

$$
w_{1}(x, a)=w_{0}(x) \frac{1+a x}{\left(1+a^{2}+2 a x\right)} \quad, \quad|a| \leq 1 / 2,
$$

que representa, com a variação do parâmetro a, uma família infinita de tais funções.

Considerando a função peso acima, Ullman prova a seguinte igualdade

$$
z \exp \left(\frac{-1}{\mu_{0}} \sum_{k=1}^{\infty} \frac{\mu_{k}}{k z^{k}}\right)=\frac{1}{2}\left(z+\left(z^{2}-1\right)^{1 / 2}+a\right),|z|>1
$$


A utilização do método de Chebyshev (visto em (3.1)) para a a construção da quadratura de Chebyshev fornece o polinômio

$$
\begin{gathered}
P_{n}(z, w(x))=E\left\{\left(\frac{z+\left(z^{2}-1\right)^{1 / 2}+a}{2}\right)^{n}\right\} \\
=\left(\frac{z+\left(z^{2}-1\right)^{1 / 2}+a}{2}\right)^{n}+\left(\frac{z-\left(z^{2}-1\right)^{1 / 2}+a}{2}\right)^{n}-\left(\frac{a}{2}\right)^{n} \\
=\frac{1}{2^{n-1}} T_{n}^{(a)}(z),
\end{gathered}
$$

onde $T_{n}^{(a)}(z)$ é um polinômio de grau $n$. Quando $a=0$ obtemos 0 polinômio de Chebyshev. Ullman mostra que $T_{n}^{(a)}(z)$, para cada $n$, têm zeros reais e distintos somente quando $-1 / 2 \leq a \leq 1 / 2$.

Em 1982, Franz Pehertorfer [21] também provou que a função peso

$$
w_{2}\left(x, a_{1}\right)=w_{0}(x) \frac{1}{\pi 1} \sum_{i=1}^{l} \frac{1+a_{i} x}{1+a_{i}^{2}+2 a_{i} x},\left|a_{i}\right| \leq 1 / 8,
$$

para $i=1,2, \ldots, 1$ tem propriedade $T$ no intervalo $(-1,1)$.

Uma outra familia de funçōes pesos, obtida depois por Byrd e stalla [3], é a seguinte :

$$
w_{3}(x, a)=w_{0}(x) \frac{1}{(2 a+1+x)}, a \geq 1
$$

As funçōes pesos obtidas por $w_{3}(x, a), a \geq 1$ são diferentes das obtidas por $w_{1}(x, a),|a| \leq 1 / 2$.

Föster [5], atravês de um método bastante diferente daqueles utilizados por Ullman e Byrdtatstalla, apresentou as funçōes 
pesos,

$$
\begin{aligned}
& w_{4}(x)=w_{0}(x)|x|^{-1 / 2}(1+|x|)^{1 / 2} \\
& w_{5}(x)=w_{0}(x)(1+x)^{-1 / 4}\left(\sqrt{2}+(1+x)^{1 / 2}\right)^{1 / 2} \\
& w_{6}(x)=w_{0}(x)\left(1+x^{2}\right)^{-1 / 4}\left(1+\left(1+x^{2}\right)^{1 / 2}\right)^{1 / 2},
\end{aligned}
$$

todas com propriedade $\mathrm{T}$.

Finalmente, citamos a familia de funçōes pesos devido a Yuan Xu [27]:

$$
\mathrm{w}_{7}(\mathrm{x}, a)=\mathrm{w}_{0}(\mathrm{x})(1+a \mathrm{x}), \quad|a|<0.27846 \ldots
$$

Podemos observar que todas as funções pesos citadas são do tipo :

$$
w_{i}(x)=w_{0}(x) v_{i}(x), i=1,2, \ldots, 7
$$

onde $v_{1}(x)$ pode ser uma função contendo, ou não, parâmetros e $w_{0}(x)$ é a função peso de Chebyshev.

As funções obtidas pela equação acima podem ser consideradas como uma modificação da função peso $\mathrm{w}_{0}(\mathrm{x})$. Com isso, podemos observar que mesmo as funções pesos modificadas podem ter propriedade $\mathrm{T}$, sugerindo o seguinte problema : caracterizar todas as funçōes pesos obtidas pelo produto $w_{0}(x) v_{1}(x)$ que têm propriedade $\mathrm{T}$. 
CAPÍTULO IV

FÓRMULAS DE QUADRATURA QUASE CHEBYSHEV

\subsection{Introdução}

Entre todas as funçōes pesos com propriedade $T$ conhecidas, somente a função peso $w_{0}(x)=\left(1-x^{2}\right)^{-1 / 2}$ admite uma fórmula de quadratura cujos nós podem ser dados explicitamente e, portanto, calculados facilmente. Em outros casos, o cálculo dos nós torna-se um problema muito trabalhoso, o que dificulta a aplicabilidade das correspondentes fórmulas de quadraturas.

Uma forma de contornar esse problema é relaxar a condição de precisão da fórmula de quadratura.

A função peso que admite fórmula de quadratura (1.1.1), mas com precisão dada por (1.1.4) com $\mathrm{p}=\mathrm{n}^{-1}$, ou seja,

$$
R_{n}(f)=0 \text { para todo } f \in P_{n-1}
$$

para todo $n \geq 1$, chamaremos função peso com propriedade quase $T$.

Podemos ver que para valores altos de $n$ não há diferença significativa de precisão entre as fórmulas de quadratura dadas por $(1.1 .1)$ e $(1.1 .2)$ e as dadas por $(1.1 .1)$ e $(1.1 .4)$ com $\mathrm{p}=\mathrm{n}-1$

o conjunto de funçōes com propriedade quase $T$, abrange o conjunto de funçōes com propriedade T. Portanto, a possibilidade de encontrar funçōes com propridade quase $\mathrm{T}$ pode ser bem maior 
que aquelas com propriedade $\mathrm{T}$.

Além disso, para qualquer função peso com propriedade quase $T$, os nós da fórmula de quadratura não são únicos, isto é, eles podem ser escolhidos de infinitas maneiras. Isso cria a possibilidade de encontrar-se conjuntos de nós que podem ter propriedades desejáveis, como por exemplo, a de serem facilmente explicitáveis.

\section{2 Construçāo da fórmula de Quadratura Quase Chebyshev}

Seja

$$
Q_{n}(z, w(x))=P_{n}(z, w(x))+c,
$$

onde $P_{n}(z, W(x))$ é dado por

$$
\begin{gathered}
P_{n}=E\left\{[f(z)]^{n}\right\}, \\
f(z)=\exp \left(\frac{1}{\mu_{0}} \int_{a}^{b} \ln (z-x) w(x) d x\right) \text { e c um número real }
\end{gathered}
$$

qualquer.

Utilizando as idéias do método de chebyshev para a construção das fórmulas de quadratura de chebyshev, é fácil mostrar que as raizes do polinômio $Q_{n}(z, w(x))$, são os nós da fórmula de quadratura quase Chebyshev.

Verifiquemos este fato:

Temos que

$$
Q_{n}(z, w(x))=\{f(z)\}^{n}+\bar{G}_{n}(z)
$$


sendo $\bar{G}_{n}(z)=G_{n}(z)+c$ uma função analítica para $|z|>1$, que assume o valor $c$ no infinito. Aqui $G_{n}(z)$ é a função definida em $(3.1 .3)$. Segue, então, que

$$
\begin{gathered}
\frac{Q_{n}^{\prime}(z, w(x))}{n Q_{n}(z, W(x)}=\frac{f^{\prime}(z)}{f(z)}+\frac{1}{Q_{n}(z, W(x))}\left\{\frac{\bar{G}_{n}^{\prime}(z)}{n}-\frac{f^{\prime}(z)}{f(z)} \bar{G}_{n}(z)\right\}, \\
\frac{Q_{n}^{\prime}(z, W(x))}{n Q_{n}(z, w(x))}=\frac{f^{\prime}(z)}{f(z)}+0\left[1 /\left(z^{n+1}\right)\right]
\end{gathered}
$$

Considerando a expansão de Laurent de $f^{\prime}(z) / f(z)$ obtemos

$$
\frac{Q_{n}^{\prime}(z, w(x))}{n Q_{n}(z, w(x))}=\sum_{k=0}^{n} \frac{\mu_{k} / \mu_{0}}{z^{k+1}}+O\left[1 /\left(z^{n+1}\right)\right]
$$

Por outro lado,

$$
\frac{Q_{n}^{\prime}(z, w(x))}{n Q_{n}(z, w(x))}=\frac{1}{n} \sum_{r=1}^{n} \frac{1}{z-\bar{x}_{r}}
$$

onde $\bar{x}_{r}$ são as raizes de $Q_{n}(z, w(x))$.

Expandindo o lado direito da expressão em termos de 1/z, obtemos

$$
\frac{Q_{n}^{\prime}(z, w(x))}{n Q_{n}(z, w(x))}=\sum_{k=0}^{\infty} \frac{\frac{1}{n} \sum_{r=1}^{n}\left(\bar{x}_{r}^{(n)}\right)^{k}}{z^{k+1}}
$$

Comparando os primeiros $n$ termos de $(4.2 .1)$ e $(4.2 .2)$ temos

$$
\mu_{k}=\frac{\mu_{0}}{n} \sum_{r=1}^{n}\left(\bar{x}_{r}^{(n)}\right)^{k}, \quad k=1,2, \ldots, n-1 .
$$

Isto é, 


$$
\int_{a}^{b} x^{k} w(x) d x=\frac{\mu_{0}}{n} \sum_{r=1}^{n}\left(\bar{x}_{r}^{(n)}\right)^{k} \quad k=1,2, \ldots, n-1 .
$$

Esta relação nos diz que se escolhermos $\bar{x}_{r}^{(n)}$, que são os zeros de $Q_{n}(z, w(x))$, para serem os nós da fórmula de quadratura dada por $(1.1 .1)$ e (1.1.4) com $\mathrm{p}=\mathrm{n}-1$ então ela será exata para todo polinômio de grau menor ou igual a $n-1$.

4.3 Um caso especial de função peso com Propriedade Quase $T$

Consideramos a classe de funções pesos dado por

$$
w_{8}(x, a)=w_{0}(x) \frac{1+a x}{1+a^{2}+2 a x}, \quad|a|<1 .
$$

Esta classe de funções pesos abrange a classe de funções pesos considerada por Ullman [23]. Temos, para esta classe de funçōes pesos, o seguinte resultado :

\section{Teorema 4.3.1}

$\mathrm{Na}$ fórmula de quadratura (1.1.1), com a classe de funções pesos $w_{8}(x, a), \quad 0<|a|<1$, se :

$$
\begin{gathered}
\gamma^{(n)}=\frac{\pi}{n} e \\
x_{r}^{(n)}=\frac{1-a^{2}}{2 a} U_{r}^{(n)}-\frac{1+a^{2}}{2 a}, r=1,2, \ldots, n, \text { onde }
\end{gathered}
$$




$$
U_{n+1-m}^{(n)}=t_{m}^{(n)}+\sqrt{\left(t_{m}^{(n)}\right)^{2}-1,} U_{m}^{(n)}=1 / U_{n+1-m}^{(n)},
$$

$m=1,2, \ldots[(n+1) / 2] \quad$ e $\quad t_{m}^{(n)}=\frac{\left(1+a^{2} \cos (2 m-1) \pi / n\right)}{\left(1-a^{2}\right)}$;

entāo, $R_{n}(f)=0$, para todo $f \in \mathbb{P}_{n-1}$.

\section{Prova}

No caso da função peso de Ullman, $P_{n}(z, w(x))$ era da seguinte forma

$$
\begin{array}{r}
P_{n}(z, w(x))=\left\{\frac{z+\left(z^{2}-1\right)^{1 / 2}+a}{2}\right\}^{n}+\left\{\frac{z-\left(z^{2}-1\right)^{1 / 2}+a}{2}\right\}^{n} \\
-\left(\frac{a}{2}\right)^{n} .
\end{array}
$$

Escolhendo $c=\left(\frac{a}{2}\right)^{n}$, para o polinômio $Q_{n}(z, w(x))$ dado por $Q_{n}(z, w(x))=P_{n}(z, w(x))+c$, obtemos:

$$
Q_{n}(z, w(x))=\left\{\frac{z+\left(z^{2}-1\right)^{1 / 2}+a}{2}\right\}^{n}+\left\{\frac{z-\left(z^{2}-1\right)^{1 / 2}+a}{2}\right\}^{n} .
$$

As raizes de $Q_{n}(z, w(x))$ podem ser facilmente calculadas. Colocando $Q_{n}(z, w(x))=0$, obtemos

$$
\left\{\frac{z+\left(z^{2}-1\right)^{1 / 2}+a}{2}\right\}^{n}=-\left\{\frac{z-\left(z^{2}-1\right)^{1 / 2}+a}{2}\right\}^{n} .
$$

Extraindo a raiz n - ésima e chamando

$V_{k}^{(n)}=(-1)^{1 / n}=\cos \frac{2 k-1}{n} \pi+i \operatorname{sen} \frac{2 k-1}{n} \pi, k=1,2, \ldots, n$,

podemos escrever 


$$
\left\{z+\left(z^{2}-1\right)^{1 / 2}+a\right\}=v_{k}^{(n)}\left\{z-\left(z^{2}-1\right)^{1 / 2}+a\right\}
$$

Daí,

$$
\begin{gathered}
\left(v_{k}^{(n)}+1\right)\left(z^{2}-1\right)^{1 / 2}=\left(v_{k}^{(n)}-1\right)(z+a), \\
{\left[\left(v_{k}^{(n)}+1\right)\left(z^{2}-1\right)^{1 / 2}\right]^{2}=\left[\left(v_{k}^{(n)}-1\right)(z+a)\right]^{2},} \\
{\left[\left(v_{k}^{(n)}\right)^{2}+2 v_{k}^{(n)}+1\right]\left[z^{2}-1\right]=\left[\left(v_{k}^{(n)}\right)^{2}-2 v_{k}^{(n)}+1\right]\left[z^{2}+2 a z+a^{2}\right]} \\
\text { sabendo-se que } \left.{ }^{2}+\frac{1}{v_{k}^{(n)}}\right]=2 \cos \theta_{k}, \quad \text { onde } \\
\quad\left[v_{k}^{(n)}+\frac{2 k-1}{n}, \pi k_{k}=1,2, \ldots, n\right. \text { obtemos } \\
2 z^{2}+\left[2 a\left(1-\cos \theta_{k}\right)\right] z-\left(1-a^{2}\right)-\left(1-a^{2}\right) \cos \theta_{k}=0
\end{gathered}
$$

Resolvendo a equação acima, para $0<|a|<1$, obtemos para as raizes $\bar{x}_{r}^{(n)}$,

$$
\begin{aligned}
& \bar{x}_{r}^{(n)}=\frac{1-a^{2}}{2 a} U_{r}^{(n)}-\frac{1+a^{2}}{2 a}, \quad r=1,2, \ldots, n \text { onde } \\
& U_{n+1-m}^{(n)}=t_{m}^{(n)}+\sqrt{\left(t_{m}^{(n)}\right)^{2}-1},{\underset{m}{U^{(n)}}=1 / U_{n+1-m}^{U^{(n)}}}_{m}^{(n)} \text { e } t_{m}^{(n)}=\frac{1+a^{2} \cos (2 m-1) \pi / n}{1-a^{2}}
\end{aligned}
$$


CAPÍtuLO $v$

\section{FÓRMULAS DE QUADRATURA DO TIPO CHEBYSHEV}

\section{1 Sobre Fórmulas de Quadratura do Tipo Chebyshev}

Consideremos, novamente as fórmulas de quadratura de Chebyshev

$(5.1 .1)$

$$
\int_{-1}^{+1} f(x) w(x) d x=\gamma^{(n)} \sum_{r=1}^{n} f\left(x_{r}^{(n)}\right)+R_{n}(f),
$$

onde $w(x)=1$ no intervalo $[-1,1]$ e $R_{n}(f)=0$ para $f \in \mathbb{P}_{n}$.

A determinação dos nós reais, $x_{r}^{(n)}$, nas condições acima de forma que (5.1.1) seja exata para $1, x, \ldots, x^{n}$, é possivel para $\mathrm{n}=1,2, \ldots, 7$ e $\mathrm{n}=9$. De acordo com resultado de Bernstein para $\mathrm{n}=8$ ou $\mathrm{n} \geq 10$, envolve nós complexos.

Este fato, tem levado muitos pesquisadores como por exemplo Barnhill et al [1], kahaner [16] e Gautschi e Yanagiwara [11] a considerarem fórmulas de quadratura modificadas.

Barnhill et al [1] considera a possibilidade de achar fórmulas de quadratura da forma

$(5.1 .2)$

$$
\int_{-1}^{+1} f(x) d x \approx \frac{2}{n} \sum_{r=1}^{n} f\left(x_{r}\right)
$$

onde os $x_{r}$ são encontrados de forma a serem simétricos, pertencentes ao intervalo $[-1,1]$ e a função 


$$
\sigma_{\mathrm{n}}=\sum_{\mathrm{k}=0}^{\mathrm{n}}\left[\frac{2}{\mathrm{n}} \sum_{r=1}^{\mathrm{n}} \mathrm{x}_{\mathrm{r}}^{\mathrm{k}}-\mu_{\mathrm{k}}\right]^{c}
$$

seja minima.

Aqui $\mu_{k}$ são os momentos definidos por :

$$
\mu_{k}=\int_{-1}^{+1} x^{k} d x
$$

Kahaner em [16] estudou o mesmo problema considerado por Barnhill et al [1], mas com uma condição adicional

$$
\frac{2}{n} \sum_{r=1}^{n} x_{r}^{k}-\mu_{k}=0, \quad k=1,2, \ldots, p, \quad p<n .
$$

- problema foi tratado por estes autores usando técnicas puramente numéricas, e, baseados nos seus resultados verificaram a presença de nós múltiplos nos casos onde $n=8,10$ e 11 .

Gautschi e Yanagiwara [11] através de técnicas matemáticas provaram alguns desses resultados observados por Barnhil e Kahaner e mostraram também como algumas regras de quadratura propostas por eles podem ser construídas resolvendo-se simples equações algébricas.

Consideremos os problemas propostos em [11], e apresentamos em seguida alguns resultados conseguidos por estes autores. 
Problema I

Sejam $p$ e $q$ inteiros, tais que $0 \leq p<n \leq q \leq \infty$. Determinar nós reais, $\left\{x_{r}\right\}_{r=1}^{n}$ (contidos no intervalo $\left.[-1,1]\right)$, tais que

$$
\sum_{j=p+1}^{q}\left[R_{n}\left(x^{j}\right)\right]^{2}=\text { minimo, }
$$

sujeito a

$(5.1 .4)$

$$
R_{n}\left(x^{j}\right)=0, \text { para } j=1,2, \ldots, p
$$

Problema I'

o problema I, mas com as restriçōes simétricas adicionais, isto é,

$$
x_{n+1-k}=-x_{k}, \quad k=1,2, \ldots, n \text {. }
$$

Se $q=n$, e $n \leq 7$ ou $n=9$, os problemas $I$ e $I^{\prime}$ são resolvidos pelas fórmulas clássicas de Chebyshev, que levam a função objetivo (5.1.3) para zero.

Vejamos que, no caso em que $q=\mathrm{n}$ realmente dá origem a nós múltiplos quando $n=8$ e $n \geq 10$.

$\hat{E}$ fácil ver que os momentos, $\mu$, satisfazem :

$$
\mu_{j}=\int_{-1}^{+1} x^{j} d x \quad \begin{cases}=0, & \text { para } j i m p a r \\ =2 /(j+1), & \text { para } j \text { par } .\end{cases}
$$


Teorema 5.1.1

Se a solução para o problema I é única, então ela é simétrica.

\section{Prova}

Suponhamos que os nós, $x_{k}, k=1,2, \ldots, n$, estejam ordenados, isto é,

$$
x_{1} \geq x_{2} \geq \ldots \geq x_{n}
$$

Se $x=\left[x_{1}, x_{2}, \ldots, x_{n}\right]$ satisfazendo $(5.1 .6)$ é uma solução do problema $I$, então $-x$ também é.

De fato, nem a função objetivo em (5.1.3), nem as restrições (5.1.4) são afetadas se $x$ for substituido por $-x$. o resto, $R_{n}\left(x^{k}\right)$, é o mesmo ( $k$ par), ou, muda de sinal ( $k$ ímpar).

Consequentemente, se (5.1.6) representa uma solução então $-x_{n} \geq-x_{n-1} \geq \ldots \geq-x_{1}$ também.

Mas da unicidade de solução do problema I segue que $x_{n+1-k}=-x_{k}$ para $k=1,2, \ldots, n$. Afirmamos a simetria da solução

\section{Teorema 5.1 .2}

Sejam $q=n$ e $n=8$ ou $n \geq 10$. Se as restrições (5.1.4) admitem soluções reais, então toda solução do problema I tem no mínimo um nó múltiplo.

\section{Prova}

Observamos que o problema I tem solução, e que qualquer solução deverá ocorrer num ponto finito $x_{1}=\left[x_{1}, x_{2}, \ldots, x_{n}\right] \in \mathbb{R}^{n}$. 
Se $p \geq 2$, de (5.1.4) com $j=2$, segue que $x$ fica restrito a uma esfera de raio $\left(n \mu_{2} / 2\right)^{1 / 2}$ e centro na origem. Se $p<2$ podemos argumentar que a função objetivo em (5.1.3) nunca será menor que

$$
\left[R_{n}\left(x^{2}\right)\right]^{2}=\left[(2 / n) \sum_{k=1}^{n} x_{k}^{2}-\mu_{2}\right]^{2}
$$

e assim, tende, com a norma de $x$, para o infinito.

Então, é suficiente considerar o problema I numa bola suficientemente grande em $\mathbb{R}^{n}$. A existência de uma solução segue, então, do fato de estarmos minimizando uma função contínua sobre um conjunto não vazio.

Da teoria dos multiplicadores de Lagrange, o ponto de mínimo satisfaz

$$
\frac{\partial}{\partial x_{1}}\left\{\sum_{j=p+1}^{n}\left(R_{n}\left(x^{j}\right)\right)^{2}-\sum_{j=1}^{p} \lambda_{j} R_{n}\left(x^{j}\right)\right\}=0, i=1,2, \ldots, n .
$$

$$
R_{n}\left(x^{j}\right)=0, \quad j=1,2, \ldots, p \text {. }
$$

Derivando, podemos escrever estas equaçōes mais explicitamente na forma

$$
\begin{aligned}
& \left(5.1 .7^{\prime}\right) \quad \sum_{j=p+1}^{n}\left(\frac{2}{n} \sum_{k=1}^{n} x_{k}^{j}-\mu_{j}\right) j x_{i}^{j-1}+\frac{1}{2} \sum_{j=1}^{p} \lambda_{j} j x_{1}^{j-1}=0, \quad i=1, \ldots, n, \\
& \left(5.1 .8^{\prime}\right) \quad \frac{2}{n} \sum_{k=1}^{n} x_{k}^{j}-\mu_{k}=0,
\end{aligned}
$$

Interpretemos $\left(5.1 .7^{\prime}\right)$ como um sistema linear homogêneo de n equaçōes a $\mathrm{n}$ incôgnitas $\mathrm{m}_{\mathrm{J}}$, isto é,

$$
\sum_{j=1}^{n} m_{j} x_{i}^{j-1}=0, \text { onde }
$$




$$
m_{j}=j\left(\frac{2}{n} \sum_{k=1}^{n} x_{k}^{j}-\mu_{j}\right), \quad j=p+1, \ldots, n .
$$

Nem todos os $m_{j}$ podem ser nulos, caso contrário (5.1.8') vale não somente para $j=1,2, \ldots, p$, mas também para $j=p+1, \ldots, n, 0$ que é impossivel pelo resultado de Bernstein. Logo, o determinante do sistema deverá ser nulo, isto é,

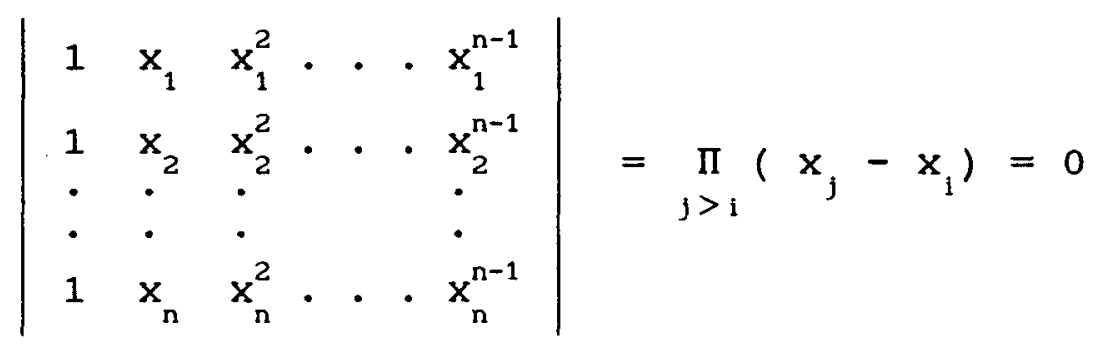

Portanto, existe no mínimo um par de indices distintos, $i, j,(i \neq j)$ para o qual $x_{1}=x_{j}$, isto é, $x_{1}$ é um nó múltiplo, o que completa a prova do teorema.

De acordo com Gautschi e Yanagiwara, é provavel que nós múltiplos ocorram também para $q>n$, mas ainda não existem provas para este fato.

Consideremos o problema $I^{\prime}$ para $q=n$. Em vista de $(5.1 .5)$ existem somente [n/2] variáveis independentes, que supomos em ordem decrescente

$$
x_{1} \geq x_{2} \geq \ldots \geq x_{v} \geq 0, \quad v=[n / 2]
$$

Se $n$ for ímpar, um nó, $x_{v+1}$, deve se anular e portanto, não aparecerá entre as variáveis em (5.1.9).

Fazendo $\pi=[p / 2]$, o problema é então equivalente à 
$(5.1 .10)$

$$
j=\sum_{\pi+1}^{v}\left[R_{n}\left(x^{2 j}\right)\right]^{2}=\text { minimo, }
$$

$$
R_{n}\left(x^{2 j}\right)=0, j=1,2, \ldots, \pi,
$$

desde que $R_{n}\left(x^{j}\right)=0$ para $j$ ímpar.

Supomos $\pi<v$ para assegurar que a função objetivo existe em (5.1.10). (Se $\mathrm{n}$ é par, isto é sempre satisfeito.) Mais explicitamente, (5.1.10) pode ser escrito da seguinte forma

$\left(5.1 \cdot 10^{\prime}\right)$

$$
\sum_{j=\pi}^{v}\left(\frac{4}{n} \sum_{k=1}^{v} x_{k}^{2 j}-\mu_{2 j}\right)^{2}=\min
$$

sujeito à

$$
\frac{4}{n} \sum_{k=1}^{v} x_{k}^{2 j}-\mu_{2 j}=0, \quad j=1,2, \ldots, \pi \text {. }
$$

Teorema 5.1 .3

Se $q=n, n=8$ ou $n \geq 10$ e se as restrições em (5.1.10') admitem soluções reais, então toda solução para o problema I' tem no mínimo, um nó múltiplo.

Prova

A existência de uma solução para o problema $I^{\prime}$ segue como a prova do teorema anterior. Se $x_{v}=0$, então a origem é um nó múltiplo, desde que os nós ocorrem em pares se n é par e, inclui um nó extra, $x_{v+1}=0$, se $n$ é ímpar. Por outro lado, qualquer solução com $x_{v}>0$, pela teoria dos multiplicadores de Lagrange, deverá satisfazer 


$$
\sum_{j=\frac{\pi}{\pi}+1}^{v}\left(\frac{4}{n} \sum_{k=1}^{v} x_{k}^{2 j}-\mu_{2 j}\right) j x_{1}^{2 j-1}+\frac{1}{2} \sum_{j=1}^{\pi} \lambda, j x_{1}^{2 j-1}=0, i=1, \ldots, v,
$$

ou seja, a matriz do sistema de equações será

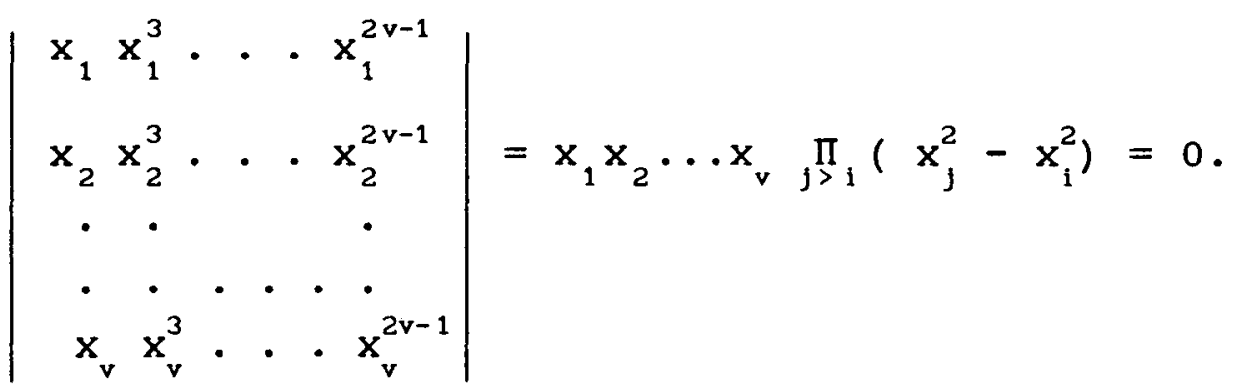

Desde que $x_{v}>0$, concluimos que $x_{1}^{2}=x_{j}^{2}$ para algum $i \neq j$, e assim $x_{i}=x_{j}$, devido a (5.1.9), ficando assim provado o teorema.

No caso de $\pi=v-1$ (considerado também por Kahaner [16]), 0 método dos multiplicadores de Lagrange é realmente poderoso e permite-nos reduzir o problema a um problema muito mais simples de solução de no máximo $\pi+2$ equações algébricas de grau menor ou igual a $\pi$. o teorema a seguir é um passo nesta direção.

\section{Teorema 5.1.4}

Seja $q=n, n=8$ ou $n \geq 10$, e $\pi=v-1$, onde $\pi=[p / 2]$, e $v=[n / 2]$. Se o problema $I^{\prime}$ admite solução, então : Uma ou ambas condições abaixo são satisfeitas :

(A) O sistema de equações

$$
\sum_{k=1}^{\pi} t_{k}^{j}=\frac{n}{4} \mu_{2 j}, \quad j=1,2, \ldots, \pi \text {, tem soluções }
$$

não negativas. 
(B) O sistema de equações

$$
\sum_{k=1}^{\pi-1} t_{k}^{j}+2 t_{\pi}^{j}=\frac{n}{4} \mu_{2 j}, \quad j=1,2, \ldots, \pi \text {, tem }
$$

soluçōes não negativas.

Colocando, para cada $t_{k}(k=1,2, \ldots, \pi)$ que satisfaz o sistema (A)

$$
x_{k}^{*}=t_{k}^{1 / 2}, \quad k=1,2, \ldots, \pi, \quad x_{v}^{*}=0 \quad e,
$$

para cada solução $t_{k}(k=1,2, \ldots, \pi-1)$ do sistema (B)

$$
x_{k}^{*}=t_{k}^{1 / 2}, \quad k=1,2, \ldots, \pi-1, \quad x_{\pi}^{*}=x_{v}^{*}=t_{\pi}^{1 / 2}
$$

Seja $\left\{x_{k}\right\}_{k=1}^{v}$ uma permutação de $\left\{x_{k}^{*}\right\}_{k=1}^{v}$ que satisfaz $(5.1 .9)$

A solução do problema $\left(5.1 .10^{\prime}\right)$ é aquele $\left\{x_{k}\right\}_{k=1}^{v}$ que fornece - menor valor para

$$
\left\{\frac{4}{n} \sum_{k=1}^{v} x_{k}^{2 v}-\mu_{2 v}\right\}
$$

Se os nós, $x_{k}, k=1,2, \ldots, v$, derivam do caso (A), então a solução do problema $I^{\prime}$ tem um nó múltiplo na origem. Se, derivam do caso (B), então o problema $I^{\prime}$ tem um nó mútiplo diferente de zero.

\section{Prova}

Já é conhecido, da prova do teorema 5.1.3, que uma solução $x=\left[x_{1}, x_{2}, \ldots, x_{v}\right]$ do problema $\left(5.1 .10^{\prime}\right)$ deverá satisfazer (5.1.11) e às restrições em (5.1.10') .

Correspondentes aos $v(v+1) / 2$ produtos em (5.1.11) existem 
também tantas possibilidades a considerar, que em vista de (5.1.9), listaremos abaixo, em termos de $t_{k}=x_{k}^{2}$ :

$$
\begin{aligned}
& t_{v}=0 \quad(1,1) \\
& t_{v-1}=t_{v}=0 \\
& t_{1}=t_{2}=\ldots=t_{v}=0 \quad(1, v) \\
& t_{v-1}=t_{v} \quad(2,1) \\
& t_{\mathrm{v}-2}=t_{\mathrm{v}-1}=t_{\mathrm{v}} \quad(2,2) \\
& \text {............ } \\
& t_{1}=t_{2}=\ldots=t_{v-1}=t_{v} \quad(2, v-1) \\
& t_{v-2}=t_{v-1} \quad(3,1) \\
& t_{v-3}=t_{v-2}=t_{v-1} \quad(3,2) \\
& \text {............. } \ldots \\
& t_{1}=t_{2}=\ldots=t_{v-2}=t_{v-1} \quad(3, v-2) \\
& t_{1}=t_{2} \\
& (v, 1)
\end{aligned}
$$

Qualquer uma das condições $(1,1), \ldots,(1, v)$, junto com as restrições $\left(5.1 .10^{\prime}\right)$ nos fornece o sistema do caso (A).

E qualquer uma das condições $(2,1)$ até $(2, v-1)$, com as restrições $\left(5.1 .10^{\prime}\right)$ dá o sistema do caso (B).

As condiçōes do grupo 3 são ou permutações daquelas do grupo 2 ou iguais a elas se $t_{v-1}=t_{v}$. Argumentos análogos são aplicados aos outros grupos.

Vemos, assim que, se $x=\left[x_{1}, x_{2}, \ldots, x_{v}\right]$ é uma solução de (5.1.10') satisfazendo (5.1.9), então $\circ$ correspondente $t=\left[t_{1}, t_{2}, \ldots, t_{v}\right]$ (com $t_{k}=x_{k}^{2}$ ) é tal que, ou $t_{v}=0$ e as componentes restantes são solução do sistema do caso (A), ou 
existe um vetor $t^{*}=\left[t_{1}^{*}, t_{2}^{*}, \ldots, t_{v-1}^{*}, t_{v}^{*}\right]$, permutação de $t$, tal que $t_{v-1}^{*}=t_{v}^{*}, e t_{1}^{*}, t_{2}^{*}, \ldots, t_{v}^{*}$ é uma solução do sistema do caso (B).

Isto prova parte do teorema. o resto da prova do teorema segue do fato que a solução desejada deve minimizar a função objetivo $\left\{\frac{4}{n} \sum_{k=1} x_{k}-\mu_{2 v}\right\}$.

Discutiremos, a seguir um método para determinar a solução dos sistemas do teorema 5.1.4, sugerido em [11].

Seja o sistema linear algébrico

$$
\sum_{k=1}^{n} x_{k}^{j}=s_{j}, \quad j=1,2, \ldots, n .
$$

Pela Identidade de Newton, visto no cap. III temos que :

Se $\left\{x_{k}\right\}_{k=1}^{n}$ é uma solução para (5.1.12) e se

$$
\xi(x)=\prod_{k=1}^{n}\left(x-x_{k}\right)=x^{n}+a_{1} x^{n-1}+\ldots+a_{n} \text { então }
$$

$(5.1 .14)$

$$
\begin{aligned}
& a_{1}=-s_{1} \\
& a_{2}=-\frac{1}{2}\left[s_{2}+a_{1} s_{1}\right] \\
& \left.\ldots \ldots \ldots \ldots \ldots+a_{n-1} s_{1}\right] . \\
& a_{n}=-\frac{1}{n}\left[s_{n}+a_{1} s_{n-1}+\ldots \ldots+a \ldots\right.
\end{aligned}
$$

Portanto, achar a solução de (5.1.12), reduz-se a determinar a solução da equação algébrica $\xi(x)=0$.

Junto com o sistema (5.1.12) consideremos o sistema

$$
\sum_{k=1}^{n} y_{k}^{j}=s_{j}-z^{j}, j=1,2, \ldots, n,
$$

onde $z$ é um parâmetro arbitrário. 
Lema 5.1 .1

Sejam $\left\{x_{k}\right\}_{k=1}^{n},\left\{y_{k}\right\}_{k=1}^{n}$ soluções para $(5.1 .12)$ e (5.1.15), respectivamente. Se

$$
\xi(x)=\prod_{k=1}^{n}\left(x-x_{k}\right)=x^{n}+a_{1} x^{n-1}+\ldots+a_{n}
$$

$$
\eta(x)=\prod_{k=1}^{n}\left(x-y_{k}\right)=x^{n}+b_{1} x^{n-1}+\cdots+b_{n} \text {, então }
$$

$$
b_{r}=z^{r}+a_{1} z^{r-1}+\ldots+a_{r}, r=1,2, \ldots, n \text {. }
$$

\section{Prova}

Em virtude de $(5.1 .12)$ e $(5.1 .15)$, temos, para valores de $x$ grande,

$$
\begin{gathered}
\frac{\xi^{\prime}(x)}{\xi(x)}=\sum_{k=1}^{n} \frac{1}{x-x_{k}}=\sum_{j=0}^{n}\left(\frac{s,}{x^{j+1}}\right)+0\left[x^{-n-2}\right] e \\
\frac{\eta^{\prime}(x)}{\eta(x)}=\sum_{k=1}^{n} \frac{1}{x-Y_{k}}=\sum_{j=0}^{n}\left(\frac{s_{j}}{x^{j+1}}\right)-\sum_{j=1}^{n}\left(\frac{z^{j}}{x^{j+1}}\right)+0\left[x^{-n-2}\right]
\end{gathered}
$$

onde $s_{0}=n$. Logo,

$$
\frac{\eta^{\prime}(x)}{\eta(x)}=\frac{\xi^{\prime}(x)}{\xi(x)}-\left(\frac{1}{x-z}-\frac{1}{x}\right)+0\left[x^{-n-2}\right]
$$

Integrando, obtemos

$$
\eta(x)=c x \frac{\xi(x)}{x-2}\left[1+o\left(x^{-n-1}\right)\right] \text {. }
$$

Desde que $\eta(x)$ e $\xi(x)$ são polinômios mônicos, a constante c desta identidade será 1 , e teremos

$$
(x-z) \eta(x)=x \xi(x)\left[1+o\left(x^{-n-1}\right)\right] \text {. }
$$

Comparando os coeficientes das potências $x^{n}, x^{n-1}, \ldots, x$ na 
igualdade acima, encontramos

$$
a_{r}=b_{r}-2 b_{r-1}, \quad r=1,2, \ldots, n,
$$

onde $b_{0}=1$, que, quando resolvido para os b's obtem-se $(5 \cdot 1 \cdot 18)$

Lema 5.1 .2

Sejam $\left\{x_{k}\right\}_{k=1}^{n},\left\{y_{k}\right\}_{k=1}^{n}$ soluções, respectivamente, para

$(5.1 .19)$

$$
\begin{gathered}
\sum_{k=1}^{n} x_{k}^{j}=s_{j}, \\
\sum_{k=1}^{n=1} y_{k}^{j}+2 y_{n}^{j}=s_{j}, \quad j=1,2, \ldots, n, e \\
\xi(x)=\prod_{k=1}^{n}\left(x-x_{k}\right)=x^{n}+a_{1} x^{n-1}+\ldots+a_{n} .
\end{gathered}
$$

$(5.1 .20)$

Então $y_{n}$ é uma raiz da equação algébrica

$(5.1 .21)$

$$
\left(\frac{d}{d y}\right)[y \xi(y)]=0
$$

\section{Prova}

Fazendo $z=y_{n}$ no lema $(5.1 .1)$, o sistema (5.1.15) torna-se - segundo sistema em $(5.1 .19)$ e, de (5.1.17), vimos que $\eta\left(y_{n}\right)=0$, isto é, de $(5.1 .18)$,

$$
\sum_{r=0}^{n}\left(\sum_{s=0}^{r} a_{s} y_{n}^{r-s}\right) y_{n}^{n-r}=0 .
$$

Invertendo a ordem da soma acima obtemos

$$
\sum_{s=0}^{n}(n-s+1) a_{s} y_{n}^{n-s}=0 \text {, que é o resultado desejado. }
$$


Este lema sugere um método para se resolver o segundo sistema em (5.1.19). Primeiramente resolvemos (5.1.21), usando as identidades de Newton para obter $\xi(x)$.

Para cada raiz positiva $y_{n}$ de $(5.1 .21)$ resolvemos

$$
\sum_{k=1}^{n-1} y_{k}^{j}=s_{j}-2 y_{n}^{j} \quad, \quad j=1,2, \ldots, n-1 \text {. }
$$

Isto é feito usando a identidade de Newton para obter o polinômio de grau n-1 correspondente e, em seguida calculando os zeros deste polinômio.

Resultados numéricos foram obtidos segundo os procedimentos citados acima para $n=8$ e $10 \leq n \leq 19$ em [11].

Com base nestes resultados numéricos observou-se que para $\mathrm{n}=8,10,11$ e 13 o problema tem soluções reais, já para $\mathrm{n}=12$ ou $14 \leq n \leq 19$ o problema não tem soluções reais. 


\section{REFERÊNCIAS BIBLIOGRÁFICAS}

[1] BARNHILL, R., DENNIS, J., NIELSON, G. A new type of Chebyshev quadrature. Math. Comp., v.23, 1969, p.437-441.

[2] BERNSTEIN, S.N. Sur les formules de quadrature de cotes et Tchebycheff. C. R. Acad. Sci. URSS, v.14, 1937, p.179-186.

[3] BYRD, P.F., STALLA L. Chebyshev quadrature rules for a new class of weight functions. Math. Comp., v.42, n.165, 1984, p. $173-181$.

[4] CHebysheV, P.L. Sur les quadratures. J. Math Pures Appl. (2), v.19, 1874, p.19-34.

[5] FÖSTER, K. J. On weight functions admitting chebyshev quadrature. Mathematics of Computation, v.49, n.179, 1987, p. 251-258

[6] GAUSS, C.F. Disquisitiones generales circa seriem infinitam $1+((\alpha \beta) /(1 \gamma)) x+\ldots$ etc. Commentationes Societatis Regiae Scientarium Gottingensis Recentiores, 2, 1812, [Werke III, p. 123-162]

[7] GAUSS, C.F. Methodus nova integralium valores per approximationem inveniendi. commentationes Societatis Regiae Scientarium Gottingensis Recentiores 3, 1814, [Werke III p.163-196] 
[8] GAUTSCHI, $W$. Nonexistence of Chebyshev type-quadratures on infinite intervals. Math. Comp., 29, 1975, p.93-99.

[9] GAUTSCHI, W. Advances in Chebyshev quadrature formula. Lecture Notes in Math., v.506, Spring-Verlag, Berlin and New York, 1976, p.100-121.

[10] GAUTSCHI, W. A survey of Gauss-Christoffel quadrature formulae. in : Butzer, P.L., Fehér, F. (Ed) E.B. Christoffel-the influence of his work in matematics and Physical Siences, Basel, Burkhäuser Verlag 1981, p.72-147.

[11] GAUTSCHI, W., YANAGIWARA, H. On Chebyshev-type quadratures. Math. Comp., v.28, 1974, p.125-134.

[12] GERONIMUS. Ja. L. On the Chebyshev quadrature formula. Izv. Akad. Nauk. SSSR Ser. Mat., v.33, 1969, p.1182-1207.

[13] HARDY, G.H., LITTELWOOD, J.E., PÓLYA, G. Inequalities. 2d ed, Cambride University Press, 1952, p.28.

[14] JACOBI, C.G.J. Ueber gauß neue methode, die werthe der integrale nüherungsweise zu furden . J. Reine Angew. Math., 1, 1826, p.301-308.

[15] JANOVIC, L.A. A quadrature formula with equal coefficientes for a certain form of the integral. (Russian) Dokl. Akad. Nauk BSSR, 15, 1971, p.873-876. 
[16] KAHANER, D. K. Chebyshev Type Quadrature formula. Math. Comp., v.24, 1970, p.571-574.

[17] KRYLOV, V. I. Approximate calculation of integrals. Macmillan, New York, 1962

[18] KRYLOV, V. I. Mechanical quadratures with equal coefficients for the integrals $\int_{0}^{\infty} e^{-x} f(x) \quad d x \quad e \int_{-\infty}^{+\infty} e^{-x} f(x) d x$ (Russian), Dokl. Akad. Nauk BSSR, 2, 1958, P.187-192.

[19] MEHLER, F. G. Bemerkungen zur theorie der mechanischen quadraturen. J. Reine Angew. Math., 63, 1864, p.152-157.

[20] NUTFULLIN, S.N., JANOVIC L.A. Cebysev quadrature formulae with certain weight functions that depend on parameters. (Russian), Visci Akad. Navuk Bssr Ser. Fiz. Math. Navuk, 1972, p. 24-30

[21] PEHERTORFER, F. Weight functions which admit Tchebycheff quadrature. Bull. Austral. Math. Soc., v.26, 1982, p. 29-38.

[22] RADAU, R. Etude sur les formules d'approximation qui servent à calculer la valeur numérique d'une intégrale definie. J. Math. Pures Appl. (3) 6, 1880, p.283-336. 
[23] SRI RANGA, A. Another quadrature rule of highest algebraic degree of precision (Submetido)

[24] TURECKII, A. H. On the existence of Chebyshev quadrature formulas for an infinite interval. (Russian), Vesci Akad. Nauk BSSR Ser. Fiz.-Tehn. Navuk, n2, 1962, p.119-120.

[25] ULLMAN, J.L. A class of weight functions that admit Tchebycheff quadrature. Michigan Math. J., v.13, 1966, p. 417-423.

[26] WILF, H.S. The possibility of Tschebycheff quadrature on infinite intervals. Proc. Nat. Acad. Sci., USA, v.47, 1961, p.209-213.

[27] XU, Y. Weight functions for Chebyshev quadrature. Math. Comp. , 53, 189, 1989, p.297-302. 